Recepción: 05 / 11 / 2017

Aceptación: 28 / 01 / 2018

Publicación: 21 / 03 / 2018

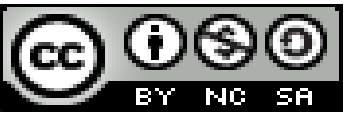

Ciencias económicas y empresariales Artículo de Revisión

\title{
Evaluación de un sistema de gestión de mantenimiento centrado en la confiabilidad (RCM)
}

\section{Evaluation of a maintenance management system focused on reliability (RCM)}

\section{Avaliação de um sistema de gerenciamento de manutenção focado na confiabilidade (RCM)}

\author{
Martín M. Ramírez-Márquez ${ }^{\text {I }}$ \\ marquez-mateo95@gmil.com \\ Paul R. Viscaino-Valencia II \\ pavisva@hotmail.com \\ Alvez R. Mera-Mosquera ${ }^{\text {III }}$ \\ meraromel@hotmail.es
}

Correspondencia: marquez-mateo95@gmil.com

\footnotetext{
${ }^{\text {I }}$ Magister en Ciencias de la Educación, Licenciado en Ciencias de la Educación Especialidad Física y Matemáticas, Ingeniero Mecánico, Técnico Superior en Mecánica Industrial, Docente de la Universidad Técnica Luís Vargas Torres de Esmeraldas, Esmeraldas, Ecuador.

II Ingeniero Mecánico, Docente de la Universidad Técnica Luís Vargas Torres de Esmeraldas, Esmeraldas, Ecuador.

III Ingeniero Mecánico, Docente de la Universidad Técnica Luís Vargas Torres de Esmeraldas, Esmeraldas, Ecuador.
} 


\section{Resumen}

El mantenimiento centrado en la confiabilidad (RCM) es una técnica que permite determinar cuáles son los procedimientos más adecuados para el óptimo funcionamiento de una empresa. A partir del mismo es posible gestionar el mantenimiento de la empresa de forma preventiva y correctiva. Bajo esta concepción, se realizó la evaluación documental del sistema de gestión de mantenimiento centrado en la confiabilidad (RCM). La metodología fue de tipo cualitativa descriptiva y documental y con un diseño no experimental. El corpus de la investigación lo constituyeron la documentación consultada acerca de las teorías e información Web en materia teórica relacionada con los RCM. Entre los resultados se pudo constatar que al momento de elegir un plan de mantenimiento del RCM dentro de una empresa, es posible adecuar la integración del grupo de las persona involucradas directamente e indirectamente en las tereas diarias, cuya apreciación de estas últimas serian indispensables debido a una posible óptica diferente con la cual se aportan los comentarios o sugerencias, permitiendo entre sus conclusiones que el uso de un plan de mantenimiento basado en la filosofía RCM, garantiza la mejora continua de los resultados de fiabilidad, disponibilidad y seguridad de una empresa, así como la reducción de los costos y el tiempo muerto para mantenimiento.

Palabras clave: sistema de gestión; mantenimiento centrado en la confiabilidad (RCM); mantenimiento prevención y mantenimiento correctivo.

\section{Abstract}

Is a technique that allows you to determine which are the most appropriate procedures for the optimal operation of a company from it, it is possible to manage preventive and corrective maintenance of the company. Under this conception, is evaluated documentary system of management $(\mathrm{RCM})$ reliability-centered maintenance. The methodology was qualitative descriptive and documentary and a non-experimental design. The corpus of the research constituted the documentation consulted about the theories and information Web in theoretical matters related to the RCM. Among the results, it was found that at the time of choosing a maintenance plan for the RCM within a company, is possible to adapt the integration of the Group of the person involved directly and indirectly in appreciate them daily, whose appreciation of these latest would be essential because of a possible different perspective with which we provide comments or suggestions, allowing among its findings that the use of RCM philosophy- 
based maintenance plans, ensures the continuous improvement of results reliability, availability and security of a company, as well as the reduction of costs and downtime for maintenance.

Keywords: management system; corrective maintenance (RCM); maintenance prevention reliability-centered maintenance.

\section{Resumo}

A manutenção focada na confiabilidade (RCM) é uma técnica que permite determinar quais são os procedimentos mais adequados para o funcionamento ideal de uma empresa. A partir disso, é possível gerenciar a manutenção da empresa de forma preventiva e corretiva. Sob essa concepção, foi realizada a avaliação documental do sistema de gerenciamento de manutenção com foco na confiabilidade (RCM). A metodologia foi qualitativa - descritiva e documental e com delineamento não experimental. $\mathrm{O}$ corpus da pesquisa foi constituído pela documentação consultada sobre as teorias e informações da Web em questão teórica relacionada ao MCR. Entre os resultados verificou-se que ao escolher um plano de manutenção do RCM dentro de uma empresa, é possível adaptar a integração do grupo de pessoas envolvidas direta e indiretamente no tareas diárias, seria necessária porque a valorização do último uma possível perspectiva diferente com os quais comentários ou sugestões, permitindo que as suas conclusões que o uso de um plano de manutenção baseado na filosofia RCM garante a melhoria contínua dos resultados de confiabilidade, disponibilidade e segurança de uma empresa são fornecidos, bem como reduzir custos e tempo de inatividade para manutenção.

Palavras chave: sistema de gestão; manutenção focada na confiabilidade (RCM); prevenção de manutenção e manutenção corretiva.

\section{Introducción}

Los comienzos del mantenimiento R.C.M datan de finales de los 50. En aquel momento el número de accidentes en la aviación mundial superaba los 60 accidentes por millón de despegues (si esto estuviera ocurriendo hoy en día, estaríamos hablando de dos accidentes diarios) y dos tercios de estos eran causados por fallas en los equipos. Con este alto índice de accidentalidad, además del gran incremento de vuelos, la industria aeronáutica tenía que ponerse manos a la obra para intentar solucionar el problema. Si la gran mayoría de accidentes estaban relacionados con fallas en los equipos, la primera solución que debían adoptar era intentar hacerlos más seguros. 
El mantenimiento, en aquellos años, equivalía a las reparaciones periódicas (se esperaba a que los componentes se gastaran después de un tiempo). La suposición que ellos hicieron era que al hacer las revisiones periódicamente, prevendrían los fallos que podían surgir. Cuando notaron que aquello no estaba funcionando, asumieron que estaban realizando demasiado tarde las reparaciones, cuando ya se apreciaba el desgaste en los equipos. Su principal idea era intentar acortar el tiempo entre reparaciones, pero los gerentes de las aerolíneas descubrieron que los porcentajes de falla no se reducían, sino que aumentaban.

Una vez que las empresas de aviación civil norteamericana se percataron que las políticas de mantenimiento existentes en aquel momento no eran las más eficientes, el primer paso que tomaron las empresas para intentar dar solución a este problema, fue la creación de unos grupos llamados "Maintenance Steering Groups" (Grupos de dirección de mantenimiento), cuya tarea era analizar el mantenimiento que sufrían los aviones en aquel momento, y estaban compuestos por representantes de las empresas fabricantes de aviones, las aerolíneas y de la Federal Aviation Administration (F.A.A, Administración Federal de Aviación). Posteriormente se desarrollaron diferentes técnicas como la técnica del RCM (Reliability Centered Maintenance). Es de indicar, que este mismo proceso de falta de mantenimiento que se genera entre las empresas activas del Cantón de Manta, les genera marcados gastos ante la poca prevención y reparaciones de sus activos por carecer de un sistema eficiente de mantenimiento. Bajo esta percepción en el presente artículo se realizó una evaluación documental, siguiente del sistema de gestión de mantenimiento centrado en la confiabilidad (RCM) que se lleva a cabo en las empresas del Cantón de Manta.

\section{Desarrollo}

La implantación de un mantenimiento centrado en fiabilidad o RCM en una planta industrial es aumentar la fiabilidad de la instalación, es decir, disminuir el tiempo de parada de planta por averías imprevistas que impidan cumplir con los planes de producción. Dicha metodología permite:

\section{Mejorar la comprensión del funcionamiento de los equipos.}

Analizar todas las posibilidades de fallo de un sistema y desarrolla mecanismos que tratan de evitarlos, ya sean producidos por causas intrínsecas al propio equipo o por actos personales.

Determinar una serie de acciones que permiten garantizar una alta disponibilidad de la planta. 


\section{Las acciones tendentes a evitar los fallos pueden ser de varios tipos:}

Determinación de tareas de mantenimiento que evitan o reducen estas averías.

Mejoras y modificaciones en la instalación.

Medidas que reducen los efectos de los fallos, en el caso de que estos no puedan evitarse.

Determinación del stock de repuesto que es deseable que permanezca en planta, como una de las medidas paliativas de las consecuencias de un fallo.

Procedimientos operativos, tanto de operación como de mantenimiento.

Planes de formación.

\section{Fases que se cumplen en un plan de mantenimiento de RCM}

El proceso de análisis de fallos e implantación de medidas preventivas atraviesa una serie de fases para cada uno de los sistemas en que puede descomponerse una planta industrial:

Fase 1: Definición clara de lo que se pretende implantando RCM. Determinación de indicadores, y valoración de éstos antes de iniciar el proceso.

Fase 2: Codificación y listado de todos los sistemas, subsistemas y equipos que componen la planta. Para ello es necesario recopilar esquemas, diagramas funcionales, diagramas lógicos, etc.

Fase 3: Estudio detallado del funcionamiento del sistema. Determinación de las especificaciones del sistema Listado de funciones primarias y secundarias del sistema en su conjunto. Listado de funciones principales y secundarias de cada subsistema.

Fase 4: Determinación de los fallos funcionales y fallos técnicos.

Fase 5: Determinación de los modos de fallo o causas de cada uno de los fallos encontrados en la fase anterior.

Fase 6: Estudio de las consecuencias de cada modo de fallo. Clasificación de los fallos en críticos, significativos, tolerables o insignificantes en función de esas consecuencias.

Fase 7: Determinación de medidas preventivas que eviten o atenúen los efectos de los fallos.

Fase 8: Agrupación de las medidas preventivas en sus diferentes categorías: Elaboración del Plan de Mantenimiento, lista de mejoras, planes de formación, procedimientos de operación y de mantenimiento, lista de repuesto que debe permanecer en stock y medidas provisionales a adoptar en caso de fallo. 
Fase 9: Puesta en marcha de las medidas preventivas.

Fase 10: Evaluación de las medidas adoptadas, mediante la valoración de los indicadores seleccionados en la fase 1.

\section{Tipos de mantenimiento.}

En la práctica existen varios tipos de mantenimiento diferenciados por la planificación, objetivos y/o necesidades. Para ello existe un plan de mantenimiento centrado en la confiabilidad (R.C.M). Las diferentes clases de mantenimiento que se puede encontrar en una industria son:

Mantenimiento ante fallo. En este caso las operaciones de mantenimiento se efectuarán tras el fallo. El objetivo principal es reparar la máquina para que vuelva a funcionar en el menor tiempo posible (se reparan o se sustituyen las piezas que han fallado). Aunque es un tipo de mantenimiento poco desarrollado, es uno de los que más se usan en la realidad (junto al correctivo).

Mantenimiento correctivo. Este tipo de mantenimiento es muy similar al anterior, ya que también actúa tras el fallo, pero se diferencia por la búsqueda del problema del fallo. Tras provocarse el fallo en la máquina se busca, diagnostica y se corrige la causa del fallo. Las ventajas e inconveniente son los mismos que en el caso anterior, a diferencia que en este tipo prevenimos la repetición del mismo fallo.

Mantenimiento preventivo. El objetivo principal del mantenimiento preventivo en prevenir el fallo en los equipos. El más empleado es el mantenimiento planificado (PPM, "Planed Preventive Maintenance"), basado en una sustitución de piezas periódica. Normalmente la sustitución se realiza independientemente del estado del estado de la pieza, donde se tiene en cuenta el número de ciclos o tiempo trabajado. La elección de los intervalos de sustitución es la parte fundamental en este mantenimiento. La ventaja de este método frente al anterior, es la planificación del mantenimiento, ya que es menos compleja al no haber tantos imprevistos y paradas no programadas.

\section{Metodología}

Se realizó una investigación con un enfoque epistémico cualitativo - descriptivo y documental y con un diseño no experimental. Hernández, Fernández y Baptista (2010) definen las investigaciones cualitativas como aquellas que permiten legitimar el conocimiento desarrollado mediante alternativas de investigación, entre estas de tipo hermenéutica que se realiza por la vía 
de la interpretación tal es este caso, posterior a la revisión de la literatura referencial acerca del plan de mantenimiento RCM. El corpus de la investigación lo constituyeron la documentación consultada acerca de las teorías científicas e información vía Web en materia teórica relacionada con los RCM. El análisis documental se hizo cotejeando la posición de diferentes autores que han escrito acerca de este tema.

\section{Análisis documental}

Posterior a la revisión de la literatura referencial acerca del plan de mantenimiento RCM, se pudo inferir lo siguiente:

Al momento de elegir un plan de mantenimiento del RCM dentro de una empresa, es posible adecuar la integración del grupo de las personas involucradas directamente y las que no estén involucradas en las tereas diarias, cuya apreciación de estas últimas serian indispensables debido a una posible óptica diferente con la cual se aportan los comentarios o sugerencias. Así después de implementar el plan es posible planificar las operaciones de mantenimientos correctivos y preventivos.

Las tareas que sean seleccionadas deben hacerse con los materiales y equipos adecuados para la seguridad, disponibilidad y costos de mantenimiento, teniendo en cuenta que lo elegido debe satisfacer aspectos fundamentales como: aplicabilidad, eficacia y economía, para ello se debe llevar un registro de todos sus equipos, planes de mantenimientos, repuestos, órdenes de trabajo, mantenimientos preventivos y correctivos y horas de paro por mantenimiento correctivo

Al considerar el mantenimiento preventivo en una empresa, es necesario el apoyo de sus proveedores, quienes pueden proporcionar información fundamental para el logro del mantenimiento planeado, de tal forma que los objetivos de ambos en cuanto a calidad, productividad y competitividad se comporten como dos líneas paralelas y de esta manera tendrán los proveedores la posibilidad de brindar respaldo con respecto a la asesoría de materiales, y adquisición de repuestos y equipos.

Fue posible constatar que el plan de mantenimiento RCM, está enfocado en los modos de fallo y en la consecución de la función del sistema, en busca de mejorar el trabajo en equipo y la cooperación a nivel de producción, siendo el resultado un rendimiento y una producción más elevado y costos de explotación más bajos. 
El plan de mantenimiento RCM, permite al personal de una empresa que haga parte de su cultura laboral o de trabajo al mantenimiento preventivo, la disponibilidad y confiabilidad del equipo, en donde la primera es definida como la probabilidad de que un equipo sea capaz de funcionar siempre que se le necesite, lo cual es lo ideal, y la segunda es definida como la probabilidad de que el equipo esté funcionando en cualquier momento que se le necesite.

\section{Conclusiones}

El diseño de un sistema de mantenimiento basado en RCM permite establecer faenas y acciones periódicas de tipo preventivas en las empresas, logrando que exista una disminución de posibles fallas y conocerlas con anticipación, para evitar las paradas de su producción y por ende la generación de costos.

Asimismo, es posible lograr coordinar todas las áreas de la empresa, de forma directa o indirecta en materia relacionada con la programación, dirección y control de su producción en pro de soluciones asertivas.

El uso de un sistema de mantenimiento basado en RCM, permite mejorar el trabajo en equipo entre las personas que interactúan en las empresas, debilitando la individualidad interna al considerar el proceso productivo o de realización de las actividades de mantenimiento de la empresa.

Se logra instaurar, ante el uso de la RCM, una cultura laboral o de trabajo al mantenimiento preventivo, el cual se lleva a cabo para asegurar la disponibilidad y confiabilidad del equipo de trabajo adscrito a una empresa.

Un plan de mantenimiento basado en la filosofía RCM, garantiza la mejora continua de los resultados de fiabilidad, disponibilidad y seguridad de una empresa, así como la reducción de los costos y el tiempo muerto para mantenimiento.

El RCM optimiza el mantenimiento a partir de la flexibilidad y la retroalimentación de la experiencia, que eliminar las tareas improductivas y que no agregan valor al mantenimiento.

\section{Referencias Bibliográficas}

Crespo, A., \& Sánchez, A. (2004). Técnicas y métodos de aplicación en la fase operativa de los equipos. Madrid: AENOR Ediciones. Díaz, 
J. (s.f.). Técnicas de mantenimiento industrial. Espinoza, F. (s.f.). Desarrollando el modelo RCM. Obtenido de slideshare: http://www.slideshare.net González, F. (2005). Teoría y práctica del mantenimiento industrial avanzado. Madrid: FC Editorial.

INDURA S.A. (2010). Manual para la recuperación y protección antidesgaste de piezas. 2M Impresores.

ITT. (2009). Manual de instalación, funcionamiento y mantenimiento.

Piroblock. (2011). Manual caldera de aceite térmico. Barcelona, España. SKF. (1992). Manual SKF de mantenimiento. Suecia. 